\title{
Middle Ear Surgery in Only Hearing Ears and Postoperative Hearing Rehabilitation
}

\author{
Myung Hoon Yoo, Byung Chul Kang, Hong Ju Park, and Tae Hyun Yoon \\ Department of Otolaryngology, Asan Medical Center, University of Ulsan College of Medicine, Seoul, Korea
}

Received March 11, 2014

Revised April 23, 2014

Accepted May 3, 2014

\author{
Address for correspondence \\ Tae Hyun Yoon, MD, PhD \\ Department of Otolaryngology, \\ Asan Medical Center, \\ University of Ulsan \\ College of Medicine, \\ 88 Olympic-ro 43-gil, Songpa-gu, \\ Seoul 138-736, Korea \\ Tel $+82-2-3010-3710$ \\ Fax $+82-2-489-2773$ \\ E-mail thyoon@amc.seoul.kr
}

Background and Objectives: The aim of this study was to evaluate surgical interventions and hearing rehabilitation in patients with chronic middle ear disease of only hearing ears. Subjects and Methods: Thirty-one patients with chronic middle ear disease of only hearing ears were enrolled in this retrospective study. Patients were classified into three groups according to the hearing level: groups A [pure tone audiometry (PTA) $<40$ ], B $(40 \leq \mathrm{PTA}<70)$, and C (PTA $\geq 70)$. We evaluated hearing results and patterns of auditory rehabilitation. Results: The main consideration for a surgical procedure was the presence of recurrent otorrhea and structural destruction. The reasons for surgical intervention in only hearing ears were otorrhea caused by chronic otitis media (68\%), cholesteatoma (29\%), and cholesterol granuloma (3\%). The causes of contralateral deaf ears were chronic otitis media (81\%) and sensorineural hearing loss (19\%). Although there was hearing deterioration in some patients with severe hearing loss (PTA $\geq 70$ ), all patients achieved dry ears after surgery and functional hearing using auditory rehabilitation. Hearing aids were used in most patients with moderate to moderately severe hearing loss and cochlear implants were used for auditory rehabilitation in patients with severe to profound hearing loss. Conclusions: Proper evaluation and indications for surgery in only hearing ears are important for successful eradication of inflammation and hearing preservation. Surgical interventions can achieve dry ear and enable further auditory rehabilitations using hearing aids and cochlear implantation.

Korean J Audiol 2014;18(2):54-57

KEY WORDS: Otitis media · Hearing loss · Deafness - Correction of hearing impairment . Rehabilitation.

\section{Introduction}

Middle ear surgery for chronic middle ear disease is aimed at eradicating inflammation and preventing disease recurrence. Preservation or improvement of hearing is an additional goal of middle ear surgery. However, surgery for middle ear disease of an ear that has a better hearing level than the opposite ear is challenging because of the importance of hearing preservation. Surgical management of the only hearing ear is stressful for the surgeon because there is a possibility of hearing deterioration. Conservative management, such as careful observation and hearing aid use, is one of the choices, but surgical management should be considered when there is refrac-

This is an Open Access article distributed under the terms of the Creative Commons Attribution Non-Commercial License (http:/creativecommons. org/licenses/by-nc/3.0/) which permits unrestricted non-commercial use, distribution, and reproduction in any medium, provided the original work is properly cited. tory otorrhea or a destructive lesion. There have been a few reports into surgical interventions in only hearing ears. Many surgeons favor surgical intervention for only hearing ears with cholesteatoma. ${ }^{1)}$ Sanna, et al. ${ }^{2)}$ used a questionnaire to study how surgeons treat patients who have chronic ear disease in only hearing ears. The results indicated that there is no consensus about the type of surgical treatment and surgical indications. Accordingly, the aim of this study was to evaluate surgical interventions and hearing rehabilitation in patients with chronic middle ear disease of only hearing ears.

\section{Subjects and Methods}

We retrospectively reviewed the medical records of $31 \mathrm{pa}-$ tients who underwent middle ear surgery of only hearing ears by one senior surgeon in a single tertiary care center from 1992 to 2011 . The age range of the patients was 25 to 65 years, with 
a mean of 45.3 years, with 21 female and 10 male patients. We examined the etiology of opposite deaf ears and the background of the decision making for surgery. Preoperative and postoperative pure tone and speech audiometry were checked to determine the hearing statuses of the patients. The average pure tone audiometry (PTA) was calculated from thresholds of $500 \mathrm{~Hz}, 1000 \mathrm{~Hz}, 2000 \mathrm{~Hz}$, and $3000 \mathrm{~Hz}$. A deaf ear was defined as that with an average air conduction threshold worse than $90 \mathrm{~dB}$ HL in PTA. Temporal bone computed tomography was preoperatively performed. We performed canal wallup mastoidectomy with or without tympanoplasty for most patients with chronic otitis media and canal wall-down mastoidectomy for most patients with cholesteatoma. Conservative and minimal surgical procedures were preferred in only hearing ears except for those with destructive lesions such as large cholesteatoma.

We classified the patients into three groups according to the hearing level. Group A contained patients with serviceable hearing levels-better than $40 \mathrm{~dB}$ HL-and only observation with-out other rehabilitation methods was recommended. Group B patients-with a hearing level between $40 \mathrm{~dB}$ HL and $70 \mathrm{~dB} \mathrm{HL}$-were recommended to use hearing aids for hearing rehabilitation. Group C contained patients with hearing levels worse than $70 \mathrm{~dB}$ HL. In addition, changes in hearing levels were also classified into three groups according to the difference between the preoperative and postoperative air conduction thresholds: improved, no change, and worsened. A significant hearing level change was defined as $15 \mathrm{~dB}$.

\section{Results}

Preoperative diagnoses of chronic middle ear disease in

Table 1. Preoperative diagnosis of only hearing ears and contralateral deaf ears

\begin{tabular}{lc}
\hline \multicolumn{1}{c}{ Only hearing ear } & No. of patients (\%) \\
\hline Chronic otitis media & $21(68)$ \\
Chronic otitis media with cholesteatoma & $9(29)$ \\
Cholesterol granuloma & $1(3)$ \\
Deaf ear & \\
$\quad$ Chronic middle ear disease & $25(81)$ \\
Sensorineural hearing loss & $6(19)$ \\
\hline
\end{tabular}

only hearing ears were as follows: 21 cases $(67.7 \%)$ of chronic otitis media, 9 cases $(29.0 \%)$ of chronic otitis media with cholesteatoma, and 1 case $(3.2 \%)$ of cholesterol granuloma (Table 1). The main causes of surgery for chronic otitis media were refractory otorrhea recurring over three times during 1 year. The indication for surgery in patients with chronic otitis media with cholesteatoma and cholesterol granuloma was prevention of further destruction of surrounding structures. Before surgery, frequent dressing changes and antibiotic medication were administered according to bacteriologic cultures for preoperative management. The average hearing level of contralateral deaf ears was $97.3 \mathrm{~dB}$ [25-75\% interquartile range $(\mathrm{IQR})=91.8-101.5]$. The most common etiology of contralateral deaf ears was surgery for chronic middle ear disease in 21 cases $(67.7 \%)$. There were four cases $(12.9 \%)$ of untreated chronic middle ear disease and six cases $(19.4 \%)$ of sensorineural hearing loss.

Surgical procedures are summarized in Table 2. Tympanoplasty was performed in five cases (16.1\%) and all were of chronic otitis media. Canal wall-down mastoidectomy and tympanoplasty was performed in nine cases $(29.0 \%)$, eight of which accompanied cholesteatoma; the other case was of recurred chronic otitis media after previous surgery. The most frequently performed procedure was canal wall-up mastoidectomy with tympanoplasty, which was performed in 17 patients (54.8\%).

Preoperative air and bone conduction PTAs were $55.3 \mathrm{~dB}$ HL $(25-75 \% \mathrm{IQR}=35.8-70.8)$ and $28.0 \mathrm{~dB}$ HL $(25-75 \%$ $\mathrm{IQR}=9.2-46.7)$, respectively, and the air-bone gap was 27.4 $\mathrm{dB}(25-75 \% \mathrm{IQR}=20.0-35.8)$. Postoperative air and bone conduction PTAs were $55.0 \mathrm{~dB}$ HL (25-75\% IQR=27.5$74.2)$ and $31.3 \mathrm{~dB}$ HL $(25-75 \% \mathrm{IQR}=12.5-49.2)$, respectively, and the air-bone gap was $23.7 \mathrm{~dB}(25-75 \% \mathrm{IQR}=$ $10.0-36.7)$. Three cases $(9.7 \%)$ showed hearing improvement after surgery: all of them were for chronic otitis media and a canal wall-up procedure was performed. Four cases $(12.9 \%)$ had worsened hearing after surgery: three of them were of patients with cholesteatoma and a canal wall-down procedure was performed in three cases (Table 3 ).

Preoperative and postoperative hearing levels and hearing rehabilitation recommendations are summarized in Table 4.

Table 2. Surgical procedures performed in only hearing ears

\begin{tabular}{lcccc}
\hline & $\begin{array}{c}\text { Tympanoplasty } \\
\text { only }\end{array}$ & $\begin{array}{c}\text { Canal wall-up } \\
\text { mastoidectomy }\end{array}$ & $\begin{array}{c}\text { Canal wall-down } \\
\text { mastoidectomy }\end{array}$ & Total \\
\hline Chronic otitis media & 5 & 15 & 1 & 21 \\
Chronic otitis media with cholesteatoma & 0 & 1 & 8 & 9 \\
Cholesterol granuloma & 0 & 1 & 0 & 1 \\
\hline Total & $5(16.1 \%)$ & $17(54.8 \%)$ & $9(29.0 \%)$ & 31 \\
\hline
\end{tabular}


Table 3. Postoperative air conduction hearing level changes compared with preoperative levels

\begin{tabular}{|c|c|c|c|c|}
\hline & Improved* & No change* & Worsened* & Total \\
\hline \multicolumn{5}{|l|}{ Category of only hearing ear } \\
\hline Chronic otitis media & 3 & 17 & 1 & 21 \\
\hline Chronic otitis media with cholesteatoma & 0 & 6 & 3 & 9 \\
\hline Cholesterol granuloma & 0 & 1 & 0 & 1 \\
\hline \multicolumn{5}{|l|}{ Choice of surgical procedure } \\
\hline Tympanoplasty & 0 & 5 & 0 & 5 \\
\hline Canal wall-up mastoidectomy & 3 & 13 & 1 & 17 \\
\hline Canal wall-down mastoidectomy & 0 & 6 & 3 & 9 \\
\hline Total & 3 & 24 & 4 & 31 \\
\hline
\end{tabular}

*a significant hearing level change was defined as a 15-dB air conduction threshold difference between preoperative and postoperative measurements

Table 4. Hearing results and principles of postoperative hearing rehabilitation

\begin{tabular}{ccccc}
\hline Group & Hearing level & $\begin{array}{c}\text { No. of patients } \\
\text { preoperatively }\end{array}$ & $\begin{array}{c}\text { No. of patients } \\
\text { postoperatively }\end{array}$ & $\begin{array}{c}\text { Hearing rehabilitation } \\
\text { recommendation }\end{array}$ \\
\hline A & $<40 \mathrm{~dB} \mathrm{HL}$ & 9 & 11 & None \\
B & $40-70 \mathrm{~dB} \mathrm{HL}$ & 14 & 12 & Hearing aid \\
C & $>70 \mathrm{~dB} \mathrm{HL}$ & 8 & 8 & Cochlear implant \\
\hline
\end{tabular}

Table 5. Results of hearing rehabilitation and changes in speech discrimination scores

\begin{tabular}{clcccc}
\hline \multirow{2}{*}{ Postop group } & \multirow{2}{*}{ Type of hearing rehabilitation } & \multirow{2}{*}{ No. of patients } & \multicolumn{3}{c}{ Mean speech discrimination score (\%) } \\
\cline { 4 - 6 } & None & 11 & Preoperative & Postoperative & After rehabilitation \\
\hline B & Hearing aid & 9 & 99.6 & 100.0 & - \\
& None & 3 & 96.4 & 96.9 & 98.7 \\
C & Cochlear implant & 3 & 96.0 & 94.7 & - \\
& Hearing aid & 3 & 61.3 & 10.7 & 50.7 \\
& None & 2 & 80.0 & 76.0 & 88.0 \\
\hline
\end{tabular}

Postop: postoperative

Nine preoperative patients belonged to group A and eight of these were still group A after surgery. They did not need any hearing rehabilitation. One patient had hearing deterioration after surgery (to group B). Fourteen patients were preoperatively classified as group B, and three of them had hearing improvement after surgery (to group A). A total of 12 patients of postoperative group $\mathrm{B}$ were recommended to use hearing aids. All eight preoperative group $\mathrm{C}$ patients were still group $\mathrm{C}$ after surgery, and they were potential candidates for cochlear implantation because of bilateral severe or profound hearing loss.

The speech discrimination scores of patients improved with hearing rehabilitation (Table 5). Postoperative group A did not require hearing rehabilitation. Postoperative group B patients were recommended to use hearing aids. We recommended hearing aids to 12 patients; although three of them refused to use these devices, the other nine patients wore hearing aids with successful rehabilitation. Speech discrimination scores improved with hearing aids. Postoperative group $\mathrm{C}$ was rec- ommended to undergo cochlear implantation: three of them underwent cochlear implantation, three of refused these implants and wore hearing aids, and the remaining two patients refused hearing rehabilitation. The patients who underwent cochlear implantation showed extremely poor preoperative (precochlear implantation) discrimination scores, with a mean value of $10.7 \%$. A clear benefit was seen after cochlear implantation, with discrimination scores improved to $50.7 \%$. Patients who wore hearing aids showed an improvement in speech discrimination scores, from $76.0 \%$ to $88.0 \%$.

During the follow-up period over 1 year, recurrent tympanic membrane perforation was identified in two patients. Myringoplasty was done in these two cases. There was no recurrent otorrhea to disturb the use of hearing aids. As a result, all patients achieved dry ears after surgery.

\section{Discussion}

Surgical management of only hearing ears remains chal- 
lenging because of a possible aggravation of hearing after surgery. Control of disease and preservation of hearing are both important in this situation. Especially in cholesteatoma, in the aspect of cholesteatoma itself, it needs to be eliminated by appropriate surgery. However in the aspect of hearing, the removal of cholesteatoma has higher risk of hearing aggravation when compared to non-cholesteatoma chronic otitis media. In our study, 3 patients had hearing aggravation and 6 patients showed no change of hearing after surgery among 9 cholesteatoma patients. But considering the growth of cholesteatoma and high possibility of hearing aggravation due to cholesteatoma, just observing the patient without surgery could not be a proper management option, even in only hearing ear.

However, Sakagami, et al. ${ }^{3)}$ reported via long-term followup results of chronic otitis media that the hearing of ears with chronic otitis media worsened significantly more than that of normal contralateral ears. Wait and see is not a good policy for only hearing ears with chronic ear disease because hearing impairments will be progressive, especially when the otorrhea is recurrent or persistent.

Surgeons should carefully choose the surgical procedure, and either canal wall-up or canal wall-down mastoidectomy procedures or tympanoplasty can be used for chronic middle ear disease in only hearing ears. The type of mastoid surgery has been shown not to affect the hearing results of chronic otitis media patients. ${ }^{4)}$ Some previously published reports remarked on the type of surgery. Yamamoto, et al. . $^{5}$ performed canal wallup mastoidectomy with tympanoplasty in 16 of 30 patients in 1997. Perez de Tagle, et al. ${ }^{6}$ performed canal wall-down mastoidectomy with tympanoplasty in 7 of 8 patients. Sakagsami, et al. ${ }^{7)}$ recommended a type 1 tympanoplasty without mastoidectomy for chronic otitis media and a canal wall-down mastoidectomy with ossicular reconstruction in patients with cholesteatoma. Battaglia, et al. ${ }^{8}$ contended that one-stage canal wall-down procedures are preferable to minimize the number of times that the ear is placed at risk.

\section{Conclusion}

All of our patients attained dry ears after surgery. Hearing aids were used in most patients with moderate to severe hearing loss and cochlear implants were used for auditory rehabilitation in patients with severe to profound hearing loss with successful results. Hence, proper preoperative evaluation and indications for surgery are important for successful eradication of inflammation and hearing preservation in only hearing ears. The association of cholesteatoma and recurrent otorrhea was a surgical indication in only hearing ears. Surgical interventions can achieve dry ears and enable further auditory rehabilitation using hearing aids and cochlear implants.

\section{REFERENCES}

1) Glasscock ME 3rd, Johnson GD, Poe DS. Surgical management of cholesteatoma in an only hearing ear. Otolaryngol Head Neck Surg 1990;102:246-50.

2) Sanna M, Shea CM, Gamoletti R, Russo A. Surgery of the 'only hearing ear' with chronic ear disease. J Laryngol Otol 1992;106:793-8.

3) Sakagami M, Maeda A, Node M, Sone M, Mishiro Y. Long-term observation on hearing change in patients with chronic otitis media. Auris Nasus Larynx 2000;27:117-20.

4) Kim MB, Choi J, Lee JK, Park JY, Chu H, Cho YS, et al. Hearing Outcomes According to the Types of Mastoidectomy: A Comparison between Canal Wall Up and Canal Wall Down Mastoidectomy. Clin Exp Otorhinolaryngol 2010;3:203-6.

5) Yamamoto E, Tasaka Y, Mizukami C, Ogata T, Okumura T, Tanabe M. Tympanoplasty on the only hearing ear with chronic otitis media. Adv Otorhinolaryngol 1997;51:35-40.

6) Perez de Tagle JR, Fenton JE, Fagan PA. Mastoid surgery in the only hearing ear. Laryngoscope 1996;106(1 Pt 1):67-70.

7) Sakagsami M, Muto T, Adachi O, Mishiro Y, Fukazawa K. Surgical management of only hearing ears with positive indications. J Laryngol Otol 2006;120:972-5.

8) Battaglia AS, Sabri AN, Jackson CG. Management of chronic otitis media in the only hearing ear. Laryngoscope 2002;112:681-5. 